\title{
K-7174 protects against OA pathogenesis through blockade of the Zmiz1-GATA axis.
}

\author{
Jiho Nam ${ }^{1}$, Li-Jung Kang ${ }^{2}$, Seok-Jung Kim³ ${ }^{3}$, Cheol Yong Choi ${ }^{4}$, Dongjun Kim ${ }^{1}$, Seong-il \\ Eyun $^{5}$, and Siyoung Yang ${ }^{6}$ \\ ${ }^{1}$ Ajou University School of Medicine and Graduate School of Medicine \\ ${ }^{2}$ Ajou University \\ ${ }^{3}$ Catholic University of Korea College of Medicine \\ ${ }^{4}$ Sungkyunkwan University \\ ${ }^{5}$ Chung-Ang University \\ ${ }^{6}$ Ajou University School of Medicine
}

November 5, 2020

\begin{abstract}
Background and Purpose Osteoarthritis (OA) is a leading cause of disability and bears a large socioeconomic cost, but we lack an effective disease-modifying therapy. There is great need to identify novel target molecules in OA pathogenesis. Here, by asking why old cartilage appears to be more sensitive to OA pathogenic factors, we identified the importance and underlying mechanism of K-7174 as a blocker of the Zmiz1-GATA axis in age-related OA pathogenesis. Experimental Approach As a novel transcriptional regulator in OA pathogenesis, Zmiz1 was isolated as an up-regulated gene from aged cartilage, using microarray and Ingenuity Pathway Analysis (IPA). Infection and intra-articular injection of Ad-Zmiz1 were performed in vitro and in vivo. Cartilage-specific Col2a1-Zmiz1 TG mice with or without DMM (destabilization of the medial meniscus) surgery were evaluated by measurement of OA manifestations. To identify a specific Zmiz1-related transcription factor and a potential inhibitor, we performed transcription factor, in silico binding, and inhibition assays. Key Results The transcriptional regulator, Zmiz1, is up-regulated in old mouse cartilage and highly expressed in damaged cartilage of OA patients and DMM mice. Col2a1Zmiz1 TG mice and DMM-induced Col2a1-Zmiz1 TG mice showed more severe cartilage destruction. Overexpression of Zmiz1 induced cartilage destruction by up-regulating Mmps and Cox2 through activation of the Zmiz1-GATA axis. Finally, we demonstrate that K-7174 interfered with the activation of the Zmiz1-GATA axis to crucially protect against OA pathogenesis and promote ECM synthesis. Conclusion and Implications The K-7174-mediated blockade of the Zmiz1-GATA axis could be a useful therapeutic strategy for blocking OA pathogenesis.
\end{abstract}

\section{Hosted file}

Main_text_BJP_v2.pdf available at https://authorea.com/users/373283/articles/491013-k-7174protects-against-oa-pathogenesis-through-blockade-of-the-zmiz1-gata-axis 
A

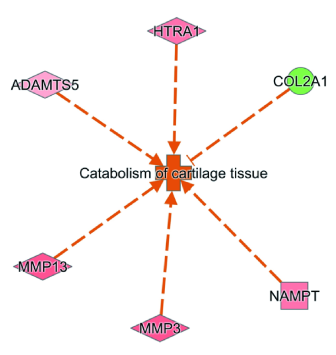

C

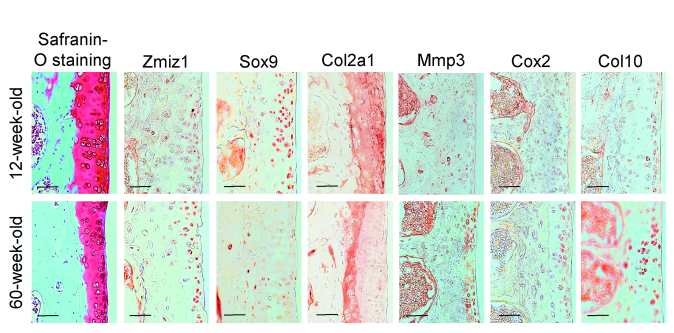

F

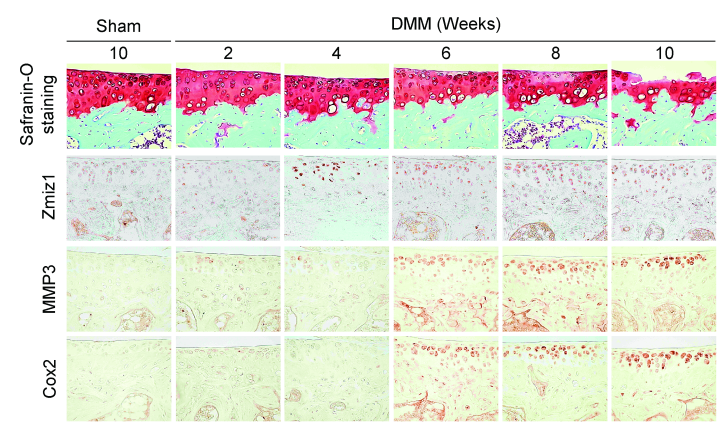

B

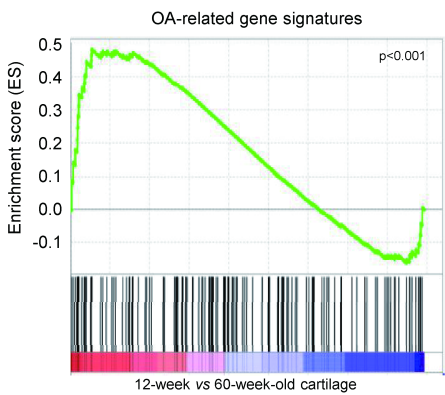

E

D

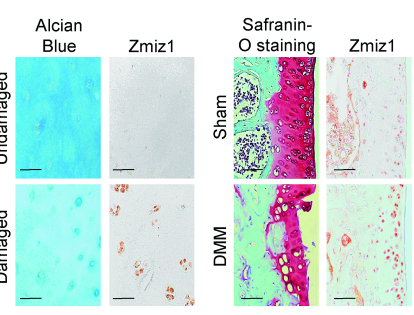

G

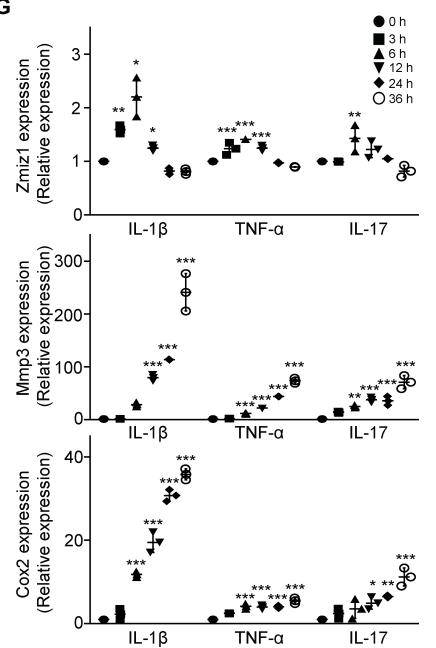


A
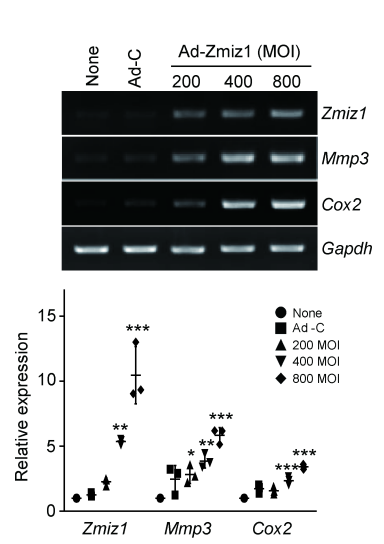

D

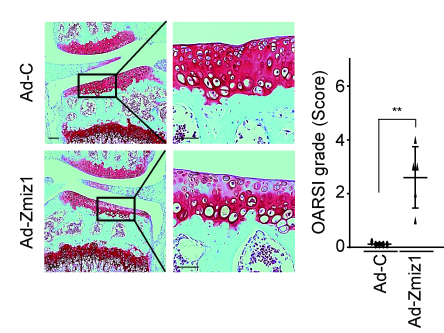

B
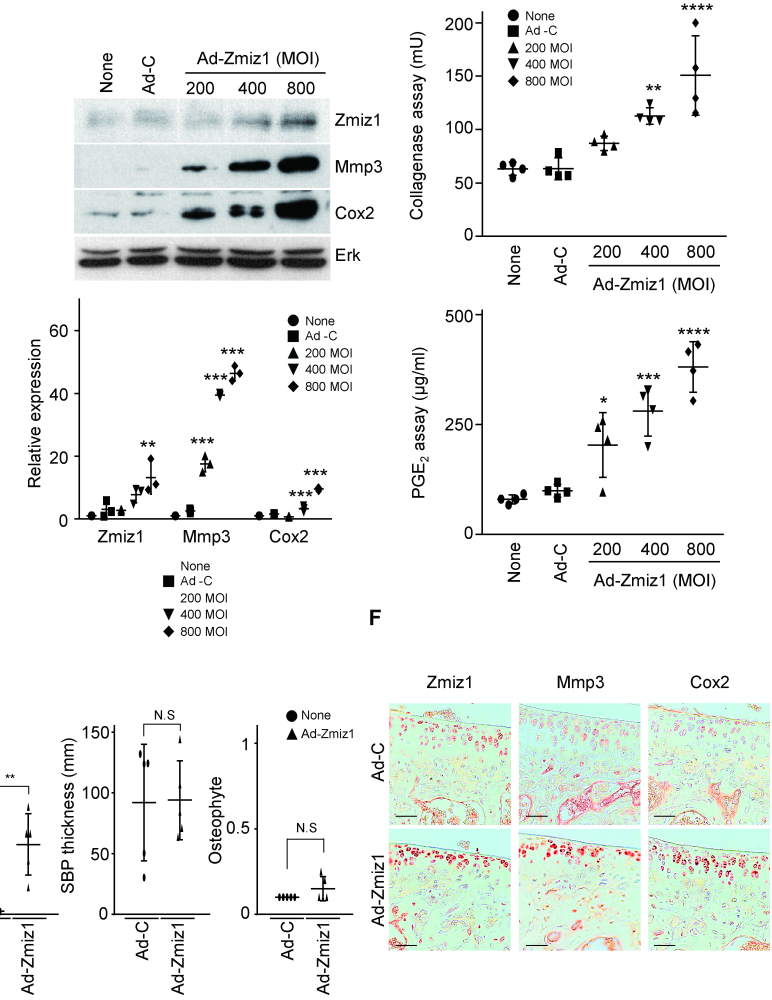
A
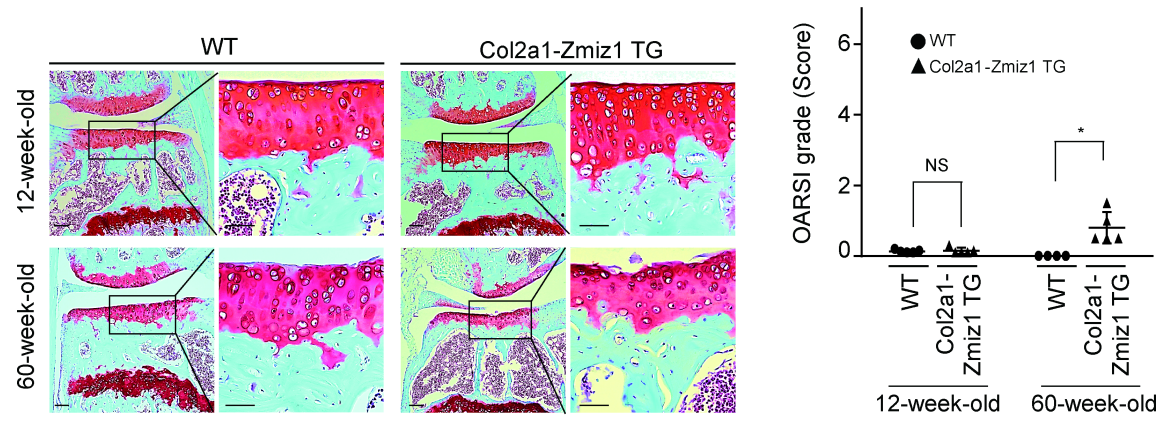

C
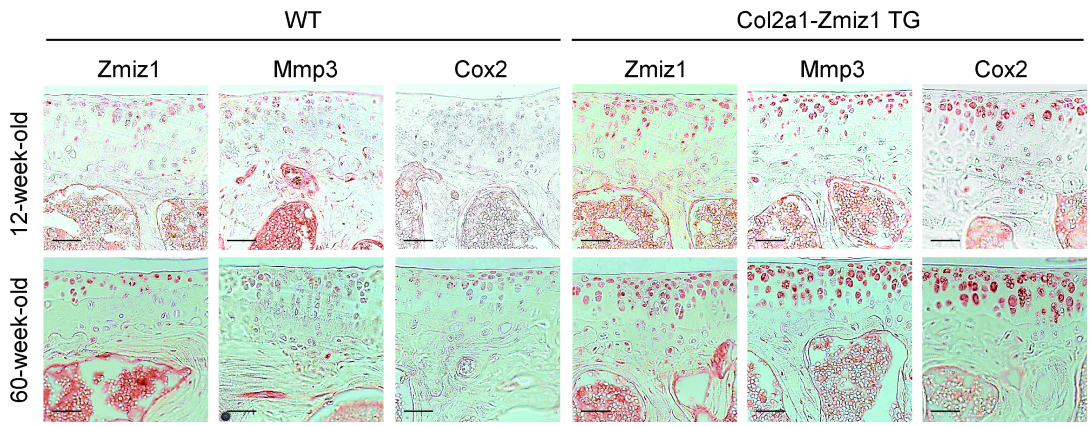
A

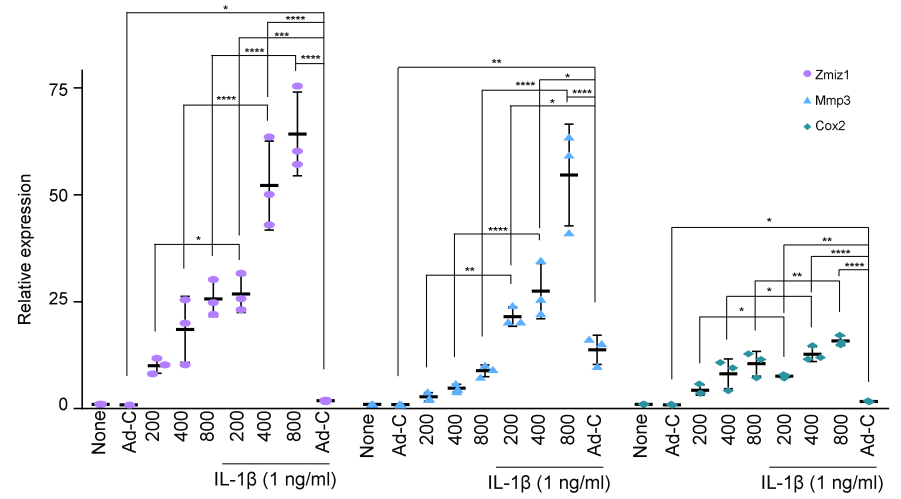

B

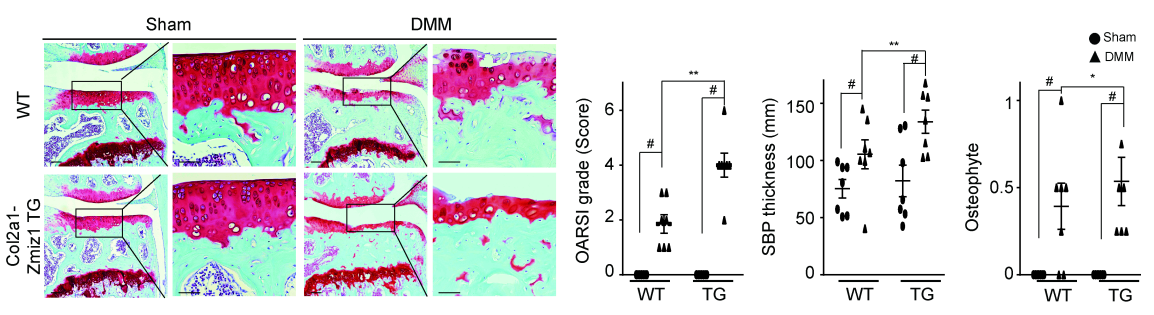

D

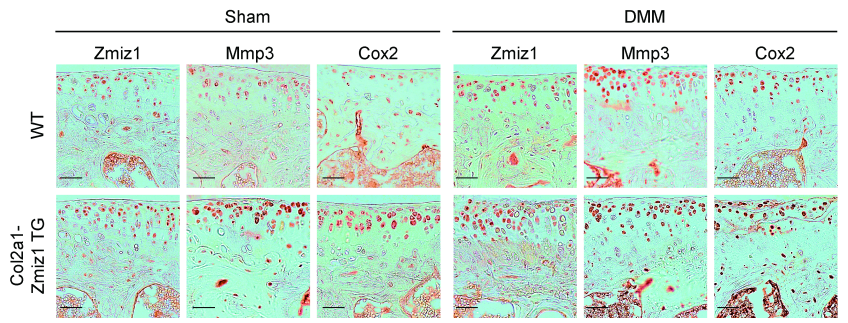


A

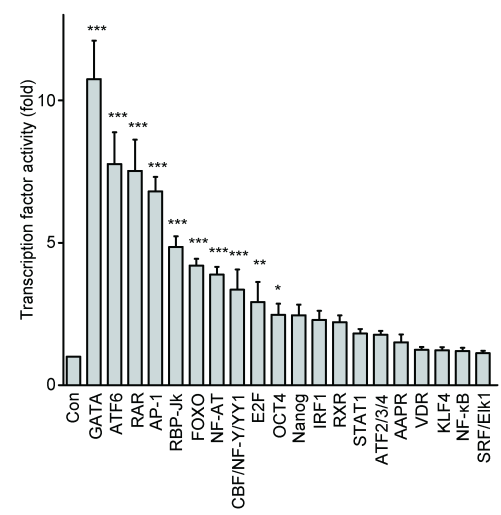

B

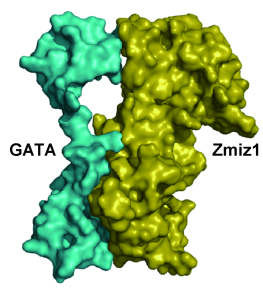

GATA vs Zmiz1 binding site

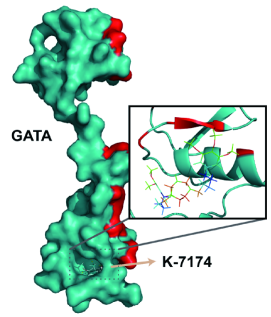

GATA vs K-7174 binding site

ARECVNCGAT ATPLWRRDRT ARECVNCGAT ATPLWRRDRT GHYLCNACGL YHKMNGQNRP GHYLCNACGL YHKMNGQNRP LIRPKKRLIV SKRAGTQCTN LIRPKKRLIV SKRAGTQCTN

CQTTTTTLWR RNASGDPVCN CQTTTTTLWR RNASGDPVCN ACGLYYKLHQ VNRPLTMRKD ACGLYYKLHQ VNRPLTMRKD $\begin{array}{ll}\text { GIQTRNRKAS GKGKKKRGSS } & \text { ACGLYYKLHQ VNRPLTMRKD } \\ \text { GIQRRKAS GKGKKKRGSS }\end{array}$

D

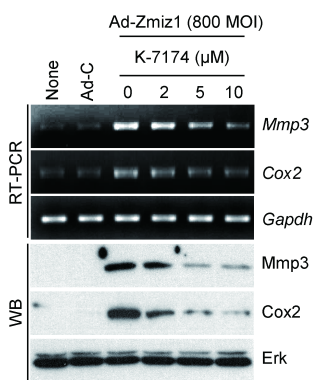

G

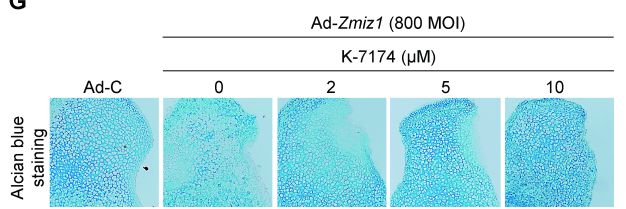

E
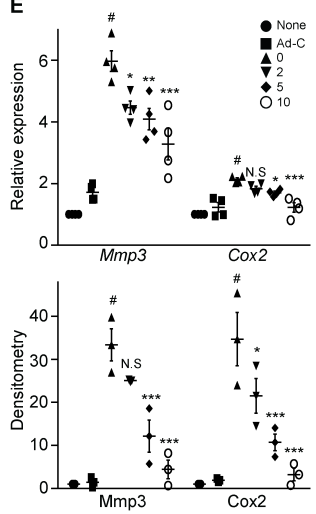

$\mathbf{F}$
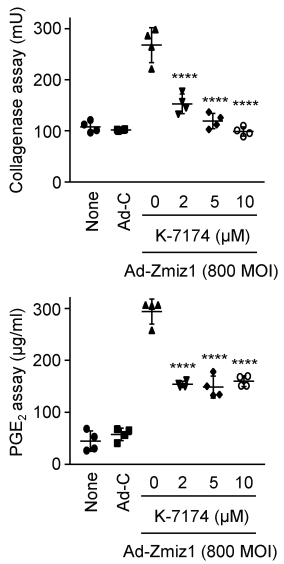


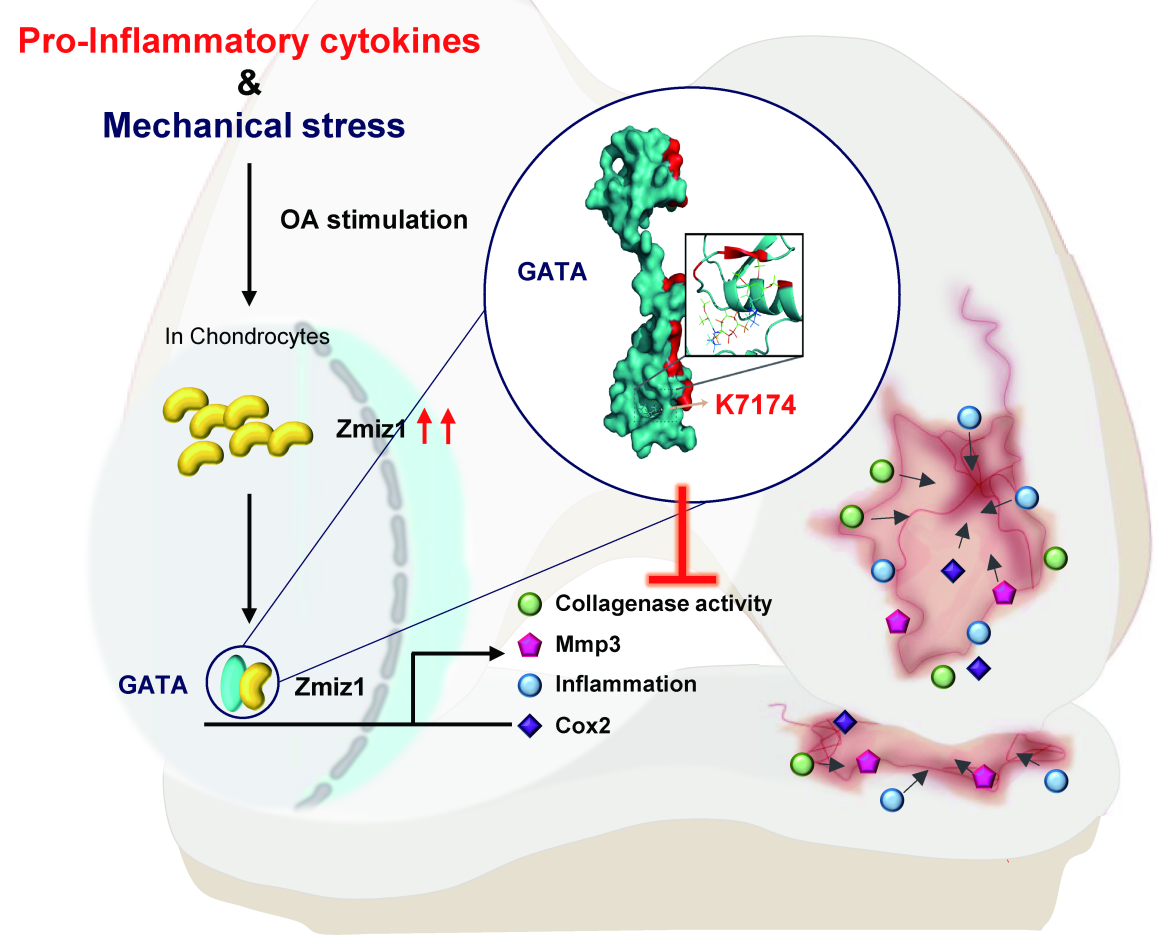

\section{Hosted file}

Table S1.pdf available at https://authorea.com/users/373283/articles/491013-k-7174-protectsagainst-oa-pathogenesis-through-blockade-of-the-zmiz1-gata-axis

\section{Hosted file}

Table S2.pdf available at https://authorea.com/users/373283/articles/491013-k-7174-protectsagainst-oa-pathogenesis-through-blockade-of-the-zmiz1-gata-axis

\section{Hosted file}

Table S3.pdf available at https://authorea.com/users/373283/articles/491013-k-7174-protectsagainst-oa-pathogenesis-through-blockade-of-the-zmiz1-gata-axis 